Ersch. in: Computational intelligence im industriellen Einsatz: Fuzzy Systeme, neuronale Netze, evolutionäre Algorithmen, Data mining ; Tagung Baden-Baden, 11. und 12. Mai $2000 /$

VDI/VDE-Gesellschaft Mess- und Automatisierungstechnik; Gesellschaft für Informatik GI. - Düsseldorf : VDI Verein Deutscher Ingenieure, 2000. - S. 21-25. - ISBN 3-18-091526-9

\title{
Visualizing High Dimensional Fuzzy Rules
}

R. Holve, M. R. Berthold, Berkeley/USA

\begin{abstract}
In this paper we present an approach to visualize a potentially high-dimensional and large number of (fuzzy) rules in two dimensions. This visualization presents the entire set of rules to the user as one coherent picture. We use a gradient descent based algorithm to generate a $2 \mathrm{D}$-view of the rule set which minimizes the error on the pair-wise fuzzy distances between all rules. This approach is superior to a simple projection and also most non-linear transformations in that it concentrates on the important feature, that is the inter-point distances. In order to make use of the uncertain nature of the underlying fuzzy rules, a new fuzzy distance-measure was developed. The visualizations of a rule set for the well-known IRIS dataset as well as fuzzy models for other benchmark data sets are illustrated and discussed.
\end{abstract}

\section{Introduction}

For the analysis of large industrial data sets, the automatic extraction of rules has raised increasing interest in the past years $[2,4]$. Two main drawbacks have inhibited the application of such algorithms to real problems. Not only does the number of extracted rules explode for many real world scenarios but in addition does the interpretability of such rules suffer tremendously in high-dimensional feature spaces, due to the high number of constraints in the rules' antecedents.

In this paper we present an approach which visualizes the entire set of fuzzy rules in two dimensions. The original fuzzy rules can be defined over a feature space of arbitrarily large dimension. Based on a new fuzzy distance between two fuzzy points in this feature space, we derive a mesh of points coupled by pairwise fuzzy distances. This matrix of fuzzy distances is then transformed into a two dimensional model, where the pairwise distances are maintained as closely as possible. This is achieve through a random initialization and a gradient descent based strategy which updates positions of all points subsequently.

\footnotetext{
*M. Berthold was supported by DFG grant Be1740/7-1.
} 
This work is a fuzzy variant of multi dimensional scaling [5] and spring embedding [8] and is similar to KOAN [7], a tool that transforms crisp distance matrices into two (or more) dimensions.

\section{$2 \quad$ Fuzzy Multidimensional Scaling}

The proposed method is based on a set of fuzzy rules $\mathbf{R}=\left\{\mathcal{R}_{j} \mid 1 \leq j \leq r\right\}$ which are defined through a membership function $\mu_{\mathcal{R}_{j}}: \mathbb{R}^{n} \rightarrow[0,1]$ in an $n$-dimensional feature space.

The $n$-dimensional set of rules is then transformed into a set of points in two dimensions: $\boldsymbol{p}_{j}=\left(p_{x}, p_{y}\right)(1 \leq j \leq r)$, where each point $p_{j}$ represents one rule $\mathcal{R}_{j}$. The goal of the procedure is to find a setup which minimizes some error function between the pairwise difference of points $\boldsymbol{p}_{i}, \boldsymbol{p}_{j}$ and the corresponding difference between the two fuzzy rules $\mathcal{R}_{i}$ and $\mathcal{R}_{j}$.

Such an algorithm exists for points in a high dimensional feature space. The typical error function known from Multi Dimensional Scaling then simply uses crisp distances:

$$
\sum_{i} \sum_{j}\left(d\left(\boldsymbol{P}_{i}, \boldsymbol{P}_{j}\right)-d\left(\boldsymbol{p}_{j}, \boldsymbol{p}_{j^{\prime}}\right)\right)^{2}
$$

for crisp points $\boldsymbol{P}_{i}, \boldsymbol{P}_{j} \in \mathbb{R}^{n}$ and an Euclidean distance function $d(\cdot)$.

In the scenario discussed here no crisp points are available but instead each fuzzy rule can be described through an imprecise region, or fuzzy point. In order to use equation 1 accordingly a fuzzy distance function is needed. We propose to use a fuzzy distance function $\tilde{d}\left(\mathcal{R}_{i}, \mathcal{R}_{j}\right)$ which generates a fuzzy interval describing the distance between two fuzzy rules $\mathcal{R}_{i}$ and $\mathcal{R}_{j}$. This fuzzy distance is completely defined through the following membership function:

$$
\begin{aligned}
& \mu_{\tilde{d}\left(\tilde{\mathcal{R}}_{i}, \tilde{\mathcal{R}}_{j}\right)}: \mathbb{R} \rightarrow[0,1] \\
& \forall d \in \mathbb{R}: \mu_{\tilde{d}\left(\mathcal{R}_{i}, \boldsymbol{R}_{j}\right)}(d)=\max _{\boldsymbol{x}, \boldsymbol{y} \in \mathbb{R}^{n}}\left\{\min \left\{\mu_{\mathcal{R}_{\boldsymbol{i}}}(\boldsymbol{x}), \mu_{\mathcal{R}_{\boldsymbol{j}}}(\boldsymbol{y})\right\}: \operatorname{dist}(\boldsymbol{x}, \boldsymbol{y})=d\right\}
\end{aligned}
$$

using some crisp distance function $\operatorname{dist}(\cdot)$ in $\mathbb{R}^{n}$ which will usually be the Euclidean distance. It can be shown that this fuzzy distance does not violate the triangular inequality.

Using the fuzzy distance in equation 2 we can again generate pairwise distances for all fuzzy rules in the given set of rules $\mathbf{R}$ and compute an error function:

$$
\tilde{E}(i, j)=\left(\tilde{d}\left(\mathcal{R}_{i}, \mathcal{R}_{j}\right)-d\left(\boldsymbol{p}_{i}, \boldsymbol{p}_{j}\right) \mid\right)^{2}
$$



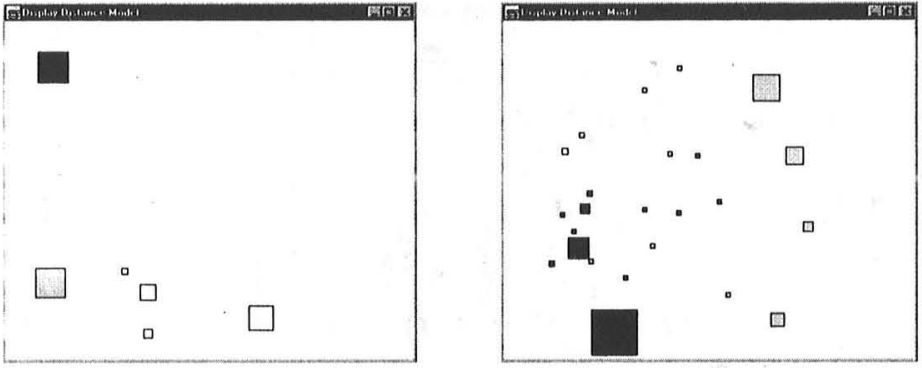

Figure 1: Visualizations of two rule sets: the well known Iris data (left) and the Breast data set (right)..

which results in a fuzzy interval as well.

Similar to classical multidimensional scaling the corresonding points in the two-dimensional representation are initially distributed randomly. The algorithm then subsequently updates the positions of all points $\boldsymbol{p}_{j}$ until a certain error minimum for the sum of the squares of all pairwise errors is reached. The update is done in small steps, using a predefined step-rate $\beta$ :

$$
\forall j: \tilde{\Delta} \boldsymbol{p}_{j}=\beta \cdot \sum_{i=1}^{|\mathbf{R}|}\left(\tilde{E}(i, j) \cdot\left(\boldsymbol{p}_{i}-\boldsymbol{p}_{j}\right)\right)
$$

which is still a fuzzy interval. In order to convert this to a crisp update value, the center of gravity of the fuzzy errors is used in equation 4 :

$$
\forall j: \Delta \boldsymbol{p}_{j}=\beta \cdot \sum_{i=1}^{|\mathbf{R}|}\left(\frac{\int_{x \in \mathbb{R}} \tilde{E}(i, j)(x) \cdot x}{\int_{x \in \mathbb{R}} \tilde{E}(i, j)(x)} \cdot\left(\boldsymbol{p}_{i}-\boldsymbol{p}_{j}\right)\right)
$$

\section{Results}

Results for two well known datasets are shown in Figure 1. On the left the Iris data [3] (4 dimensional feature space) is depicted, the right shows one benchmark of the Statlog collection [6], the Breast data set (9 features). Rules of different class (Iris: 3 , Breast: 2 ) are shown in different grey scales. The size of each rectangle illustrates the importance of a rule, which in this case is determined by the number of covered training examples.

It is interesting to see how for the Iris data one class (dark rectangle, Iris Setosa) is clearly separable from the other two. But also for the other two classes, only two rules of low importance (indicated by the smaller size) are close to each other. This is coherent with the generalization capability of the shown rule set, which achieves close to $95 \%$ correct 


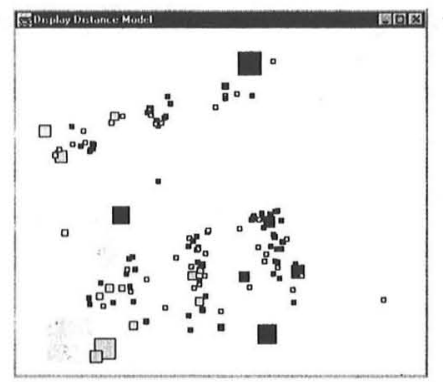

Figure 2: Visualization of the rule set for the Australian Credit data. .

classification on unseen test data. The rule set for the Breast data also shows nice separability for the most important rules of the two classes, and only a small number of rules with lower relevance lie in an area of overlap. Also this rule set achieves a generalization capability of roughly $90 \%$.

Figure 2 shows a second data set from the StatLog archive, the Australia Credit data. Here two classes need to be distinguished and the fuzzy learning algorithm generates 132 rules, 70 for class 1 and 62 for class 2 . It is interesting to see how the rules are grouped into two main cluster (top left, bottom center) with three rules in between these cluster. The larger rules are easily separable from rules of conflicting class but a large number of smaller rules are mixed with rules of the other class, indicating a larger area of possible confusion and hence probably an overall larger generalization error. Tests on unseen data validate this assumption, the classification accuracy on the unseen data set lies under $85 \%$. In the context of explorative data analysis it would be particularly interesting to focus on the two clusters of rules individually and also investigate some outliers, such as the three rules in between the two cluster and the one rule of class 2 which is far away from any other rules (bottom right). Our current implementation does not (yet) support such interaction, however.

\section{Conclusions}

In this paper a methodology was presented to visualize an entire set of fuzzy rules from a high dimensional feature space. The visualization maintains the pair-wise distances between the fuzzy rules as much as possible and gives therefore insights into the organization of the rule set in the original feature space. The presented methodology not only provides a way to visualize the entire model at once but also promises interesting opportunities awh 
for user interaction with the entire model and hence offers an interesting addition to intelligent data analysis [1] in the area of model exploration.

\section{Acknowledgments}

M. Berthold was supported by stipend Be1740/7-1 of the "Deutsche Forschungsgemeinschaft" (DFG). The authors thank Prof. Lotfi A. Zadeh and his Berkeley Initiative in Soft Computing (BISC) for providing office space and the opportunity for stimulating discussions.

\section{Literature}

[1] M. Berthold and D. J. Hand, editors. Intelligent Data Analysis: An Introduction. Springer Verlag, 1999.

[2] M. R. Berthold and K.-P. Huber. Constructing fuzzy graphs from examples. Intelligent Data Analysis, 3(1):37-54, 1999. (http://www.elsevier.nl/locate/ida).

[3] R. A. Fisher. The use of multiple measurements in taxonomic problems. In Annual Eugenics, II, 7, pages 179-188. John Wiley, NY; 1950.

[4] R. Holve. Investigation of automatic rule generation for hierarchical fuzzy systems. In Proceedings of FUZZ IEEE at 1998 IEEE World Congress On Computational Intelligence, pages 973-978, Anchorage, Alaska, May 1998. IEEE Press.

[5] J. Meulman. A distance approach to nonlinear multivariate analysis. DSWO Press, Leiden, The Netherlands, 1986.

[6] D. Michie, D. J. Spiegelhalter, and C. C. Taylor, editors. Machine Learning, Neural and Statistical Classification. Ellis Horwood Limited, 1994.

[7] L. Pfefferer. Objektzentrierte Visualisierung mehrdimensionaler Daten als Erweiterung konventioneller Datenbankmodelle (Phd Thesis). Herbert Utz Verlag, Munich, 1996.

[8] N. R. Quinn and M. A. Breuer. A force directed component placement procedure for printed circuit boards. IEEE Transactions on Circuits and Systems, CAS-26(6):377$388,1979$. 